\title{
Direct affinity of dopamine to lipid membranes investigated by Nuclear Magnetic Resonance spectroscopy
}

\author{
Yashasvi Matam, Bruce D. Ray, and Horia I. Petrache* \\ Department of Physics, Indiana University Purdue University Indianapolis, Indianapolis, IN \\ 46202
}

\begin{abstract}
Dopamine, a naturally occurring neurotransmitter, plays an important role in the brain's reward system and acts on sensory receptors in the brain. Neurotransmitters are contained in lipid membraned vesicles and are released by exocytosis. All neurotransmitters interact with transport and receptor proteins in glial cells, on neuronal dendrites, and at the axonal button, and also must interact with membrane lipids. However, the extent of direct interaction between lipid membranes in the absence of receptors and transport proteins has not been extensively investigated. In this report, we use UV and NMR spectroscopy to determine the affinity and the orientation of dopamine interacting with lipid vesicles made of either phosphatidylcholine (PC) or phosphatidylserine (PS) lipids which are primary lipid components of synaptic vesicles. We quantify the interaction of dopamine's aromatic ring with lipid membranes using our newly developed method that involves reference spectra in hydrophobic environments. Our measurements show that dopamine interacts with lipid membranes primarily through the aromatic side opposite to the hydroxyl groups, with this aromatic side penetrating deeper into the hydrophobic region of the membrane. Since dopamine's activity involves its release into extracellular space, we have used our method to also investigate dopamine's release from lipid vesicles. We find that dopamine trapped inside PC and PS vesicles is released into the external solution despite its affinity to membranes. This result suggests that dopamine's interaction with lipid membranes is complex and involves both binding as well as permeation through lipid bilayers, a combination that could be an effective trigger for apoptosis of dopamine-generating cells.
\end{abstract}

This is the author's manuscript of the article published in final edited form as: Matam, Y., Ray, B. D., \& Petrache, H. I. (2016). Direct affinity of dopamine to lipid membranes investigated by Nuclear Magnetic Resonance spectroscopy. Neuroscience Letters, 618, 104-109. http://doi.org/10.1016/j.neulet.2016.02.052 
Keywords: UV spectroscopy, NMR spectroscopy, dopamine, phosphatidylcholine, phosphatidylserine

* Corresponding author; e-mail: hpetrach@,iupui.edu

\section{HIGHLIGHTS}

- NMR analysis on dopamine was performed to determine the affinity and orientation of the neurotransmitter interacting with lipid vesicles.

- Dopamine has a higher affinity to PS lipid vesicles than to PC.

- Although dopamine has a high affinity for lipid headgroups it can also permeate lipid bilayers.

- Results suggest that dopamine-PS lipid interactions could affect cellular apoptosis and possibly stop the proliferation of Parkinson's to other healthy and normal functioning dendrites. 


\subsection{INTRODUCTION}

Dopamine (DA) is a naturally occurring neurotransmitter responsible for controlling rewardmotivated behavior in the human body. It is essential for human life and decreased levels of dopamine are known to contribute to certain diseases, such as Parkinson's syndrome. More specifically, Parkinson's syndrome is a progressive neurodegenerative disease of dopaminergic neurons, which affects motor control and sometimes physiological behavior. It has been shown that $\alpha$-Synuclein plays an important role in the regulation of dopamine biosynthesis, acting to reduce the activity of tyrosine hydroxylase (TH) [1]. Aggregation of $\alpha$-Synuclein in neurons produce "Lewy Bodies", which cause interruptions in the transmission of signals throughout the Central Nervous System. The study suggests that overexpression of $\alpha$-Synuclein in dopaminergic cells dramatically reduces TH activity [1]. Consequently, this reduction of DA synthesis causes degeneration of dopaminergic neurons and yields Parkinson's disease.

Chemically, dopamine $\left(\mathrm{C}_{8} \mathrm{H}_{11} \mathrm{NO}_{2}\right)$ has two $\mathrm{OH}$ (hydroxyl) groups and a $\mathrm{CH}_{2}-\mathrm{CH}_{2}-\mathrm{NH}_{2}$ (ethylamine) group attached to a benzene ring. Because dopamine interacts with biochemicals inside the human nervous system, it is essential that dopamine functions at a physiological $\mathrm{pH}$ of 7. As a result, dopamine has a net positive charge [2]. Levodopa is a metabolic precursor of dopamine, which gets converted in the brain by the enzyme DOPA decarboxylase [3]. Dopamine is further metabolized to the neurotransmitters epinephrine and norepinephrine which are both essential to a properly functioning nervous system. The neurotransmitters mentioned above interact with transport and receptor proteins in glial, dendritic, and axonal button membranes. The axonal button membranes are comprised of complex mixtures of lipids, however the predominant lipid classes are the phosphatidylcholines (PC) and the phosphatidylserine (PS) lipids, depending on location [4]. PC lipids in particular are commonly found in the outer leaflet of the synaptic vesicle [5] and, although not studied extensively in DA interactions, as a subclass of lecithin it is an essential precursor to acetylcholine that regulates signaling in the brain. The main function of choline and its metabolites is to transduce membrane signals and limited data available suggest that an increase in PC supplementation can alleviate the symptoms of psychiatric disorders, such as Schizophrenia, bipolar disorder, and Alzheimer's and Parkinson's [6, 7].

As opposed to PC lipids, PS lipids are most commonly found in the inner side of the plasma membrane. However, during apoptosis, PS lipids can flip to the extracellular surface acting as a 
signal for macrophages to engulf apoptotic cells [8]. Because of the orientation of the positively charged choline and the negatively charged phosphate group and serine carboxylate, the overall charge on PS is -1, thus, a positively charged molecule would create the most effective interaction for macrophage activity.

All neurotransmitters, in some way, interact with lipid bilayers either in the vesicle or synaptic membrane. Extensive research highlights the importance of brain lipid composition interaction with neurotransmitters for a properly functioning depolarization and its effect. Without a proper interaction among the two, certain neuropsychiatric disorders can arise [9]. The aim of this paper is to understand the interaction and affinity of dopamine to PS and PC lipid vesicles using NMR and Ultraviolet/Visible (UV/Vis) Spectroscopy. Second-derivative UV spectroscopy and NMR have both been used in our laboratory to measure direct interactions between membranes and aromatic side chains of amino acid residues [10] and here we apply these methods to dopamine.

Our recently developed NMR method uses an equivalent isopropanol concentration (EIC) scale to describe the interaction of aromatic moieties with lipid membranes as demonstrated for aromatic side chains of amino acid residues [10]. In this method, a range of isopropanol concentrations is used to provide a reference scale for hydrophobic interactions. Therefore, similar UV and NMR measurements were made with dopamine dissolved into varying concentrations of isopropanol to establish a standard EIC curve for measurement of dopamine interactions with either PC or PS lipid vesicles. Our results suggest that consideration of dopamine's interactions with specific lipids could help the development of treatments of dopamine-related diseases by apoptosis of damaged neurons through biochemical means.

\subsection{MATERIALS AND METHODS}

\subsection{Materials}

Dopamine hydrochloride (99.99\% pure) and isopropanol (99.9\% pure) were purchased from Sigma-Aldrich, trimethylsilyl propanoic acid (TMSP), deuterated isopropanol, and deuterium oxide $\left(\mathrm{D}_{2} \mathrm{O}\right)$ were purchased from Cambridge Isotope Laboratories, and 1, 2-dioleoyl-sn-glycero3-phosphocholine (DOPC) and 1, 2-dioleoyl-sn-glycero-3-phosphoserine (DOPS) from Avanti Polar Lipids. 


\subsection{UV Spectroscopy}

A Varian CARY $50 \mathrm{UV} / \mathrm{Vis}$ Spectrometer was used to measure the absorbance levels of dopamine in isopropanol (IPA) solutions. The concentration of dopamine in deionized (DI) water solution used is $4 \mathrm{mM}$ in a total volume of $10 \mathrm{~mL}$ (stock solution). Solutions were made at 11 different concentrations of DA in IPA such that $150 \mu \mathrm{L} \mathrm{DA}+x+y=1 \mathrm{~mL}$ solution, where $x$ is the volume of IPA in $50 \mu \mathrm{L}$ increments $(0 \leq x \leq 500 \mu \mathrm{L})$ and $y$ is the volume of DI water required for a total volume of $1 \mathrm{~mL}$ per sample. A control (blank) sample without dopamine was also measured for reference. Samples were loaded into UV cuvettes and shaken 50 times for mixing. Each sample was tested 5 times for UV absorbance, including the blank. Wavelengths selected for the measurement ranged from 240 to $500 \mathrm{~nm}$. A Matlab program (developed in-house) was used to find the peak wavelength $\left(\lambda_{\text {peak }}\right)$.

\section{$2 .{ }^{1} H$ NMR Spectroscopy}

Single pulse ${ }^{1} \mathrm{H}$ NMR was used to conduct experiments at $500 \mathrm{MHz}$ on a Varian (Palo Alto, CA) INVOA 500 Spectrometer in a magnetic field of $11.75 \mathrm{~T}$ at $25^{\circ} \mathrm{C}$. Samples were rotated at $16 \mathrm{~Hz}$ at $6 \mathrm{kHz}$ sweep width, collecting 8,192 data points. A line broadening of $0.1 \mathrm{~Hz}$ was applied. Varian and matNMR software were used to analyze collected data. Overall, NMR samples were collected on 3 sets of samples: 1) 11 samples of DA in IPA, 2) 4 samples of DA in the presence of DOPC vesicles, and 3) 4 samples of DA in the presence of DOPS vesicles.

The first set of samples (DA in IPA) were used for construction of EIC reference curves. DA concentration was $1 \mathrm{mM}$ in a total sample volume of $700 \mu \mathrm{L}$, following the relationship $35 \mu \mathrm{L}$ DA $+5 \mu \mathrm{L}$ TMSP $+a+b=700 \mu \mathrm{L}$, where $a$ is the volume of IPA in $35 \mu \mathrm{L}$ increments $(0 \leq a \leq 350$ $\mu \mathrm{L}$ ), and $b$ is the volume of $\mathrm{D}_{2} \mathrm{O}$ in each sample.

Lipid vesicle samples were prepared at lipid:DA ratios of $0: 1,1: 3,1: 1,3: 1$, and 10:1, at a total volume of $700 \mu \mathrm{L}$ per sample. Each lipid sample was sonicated in a warm water bath for 10 minutes until the sample turned clear indicating the presence of unilamellar vesicles. The solution was then transferred into a volumetric flask and shaken 50 times before being transferred into an NMR tube. 


\subsection{DA leakage measurements}

${ }^{1} \mathrm{H}$ NMR spectroscopy was also used to detect leakage of DA at 48 hours after being trapped inside lipid vesicles. DA-containing lipid vesicles were first measured at $0 \mathrm{~h}$ and then placed on a filter for $48 \mathrm{~h}$. The solution with leaked dopamine that collected in a tube placed below the filter was then measured by NMR.

\subsection{RESULTS}

\subsection{UV/ Vis Spectroscopy}

UV absorption spectroscopy of DA/IPA solutions was performed in order to determine the effect of hydrophobic environments on the peak absorption wavelength $\left(\lambda_{\text {peak }}\right)$ of dopamine. Our measurements show no appreciable change of $\lambda_{\text {peak }}$ as a function of increasing IPA concentration (data not shown). The average $\lambda_{\text {peak }}$ for all samples was measured around $288 \mathrm{~nm}$, close to the value reported earlier in $1 \mathrm{mM}$ concentration of DA/IPA [11]. We note that an absorption in the 200 to $800 \mathrm{~nm}$ region most commonly occurs due to a transition of non-bonding valence-shell electron pairs to the $\pi^{*}$ energy level. Our measured $\lambda_{\text {peak }}$ for DA indicates that the absorbance is a result of $n \rightarrow \pi^{*}$ transition in nitrogen and this transition is insensitive to changes of solution hydrophobicity. We therefore turned to NMR spectroscopy which can provide more detailed information on dopamine behavior in hydrophobic environments as we show next.

\section{2 ${ }^{1} \mathrm{H}$ NMR Spectroscopy}

\subsubsection{Reference Spectra}

Figure 1 shows the ${ }^{1} \mathrm{H}$ NMR spectra of DA for a broad range of IPA concentrations. There are three distinct groups of resonance frequencies in the aromatic part of the spectra. In the absence of IPA, the doublet just above $6.9 \mathrm{ppm}$ corresponds to $\mathrm{H}_{\varepsilon 1}$ (see Fig. 6 for hydrogen sites). The overlapping resonances around $6.85 \mathrm{ppm}$ correspond to $\mathrm{H}_{\delta 2}$ and the last group of doublets just above $6.75 \mathrm{ppm}$ corresponds to $\mathrm{H}_{\delta 1}$. As the concentration of IPA is increased, all resonances move towards lower ppm values with some slight deviations around 25-30\% IPA which are due to experimental errors. The systematic change of chemical shifts of dopamine with increasing concentration of IPA is summarized in Fig. 2. We notice that trend lines for each resonance are well determined and do not intersect each other, a feature that allows us to describe the interaction of dopamine with lipid vesicles by using equivalent IPA concentrations. We should also mention that additional measurements were ran at $5 \mathrm{mM}$ DA to test the possibility of aromatic stacking that 
could in principle influence the interpretation of results. Test results show that aromatic stacking of dopamine is not present in our samples.

\subsubsection{Measurements with lipid vesicles and construction of EIC maps for dopamine}

Having obtained the reference curves in Fig. 2, we proceeded with measurements of dopamine in the presence of lipid vesicles. These samples contain no IPA. Figure 3 shows dopamine spectra in the presence of PC lipid vesicles at various PC:dopamine ratios while Fig. 4 shows dopamine spectra in the presence of PS lipid vesicles. For both sets of samples, the spectra indicate an interaction of dopamine with lipid vesicles as measured by changes in chemical shifts as well as peak broadening.

To describe the interaction of dopamine with lipid vesicles, we use the measured chemical shift value for each hydrogen site (resonance) to find the corresponding IPA concentration using the reference curves in Fig. 2. The equivalent isopropanol concentration (EIC) maps that we obtain for dopamine in the presence of either PC or PS lipid vesicles are shown in Fig. 5. The purpose of EIC maps is to quantify the hydrophobicity experienced by each hydrogen site of dopamine when bound to lipid membranes. We find that the chemical shifts of $\mathrm{H}_{\delta 2}$ and $\mathrm{H}_{\varepsilon 1}$ sites of dopamine in the presence of 10:1 PC lipid correspond to equivalent isopropanol concentrations of 20 and 25, respectively, while the $\mathrm{H}_{\delta 1}$ chemical shift corresponds to $\mathrm{EIC}=40$. In the presence of PS vesicles,

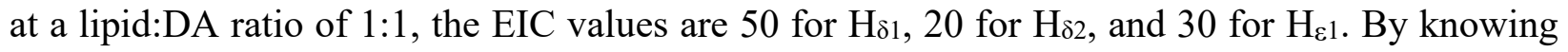
the arrangement of aromatic hydrogens and using the EIC maps it is possible to specify the orientation of dopamine when bound to the lipid bilayers. For both PC and PS lipids, the $\mathrm{H}_{\delta 1}$ and $\mathrm{H}_{\varepsilon 1}$ sites have larger EIC values indicating that this side of the aromatic ring has more affinity to the lipid membranes that the other side. This preferred orientation is depicted schematically in Fig. 6.

Since our measurements indicate a strong affinity of DA to membranes, it is interesting to see if in fact dopamine can escape (leak out) after being trapped inside lipid vesicles. When high concentrations of DA are expelled from PS lipids, it has been shown that this process can cause macrophage activity [12]. In our DA leakage experiment, the presence of dopamine inside lipid vesicles was first measured at $0 \mathrm{~h}$ and the externalized dopamine was detected by NMR after $48 \mathrm{~h}$ (see Methods). The spectra corresponding to these measurements are shown in Fig. 7 and indicate 
that DA does in fact leak out of both DOPC and DOPS vesicles. It is important to note that the $0 \mathrm{~h}$ spectra might differ from those in Figs. 3 and 4 because the vesicle solution has been washed in order to eliminate the initial amount of external DA. After 48h, the NMR spectra of filtered dopamine solution is similar to that of dopamine in water, although it is possible that some small lipid vesicles could have passed through the filters affecting the measured spectra.

\subsection{DISCUSSION}

By construction of EIC maps, we can determine the affinity of dopamine to different types of lipid vesicles and in addition, we can specify a preferential orientation when bound to membranes. In this respect, we find larger EIC values in the presence of PS than in the presence of PC lipid, which indicates a higher affinity of dopamine for the negatively charged PS lipids, as expected based on electrostatic interactions [13]. However, the orientation to dopamine when bound to membranes is similar for the two lipids since in both cases the EIC values are larger for the $\mathrm{H}_{\delta 1}$ and $\mathrm{H}_{\varepsilon 1}$ sites compared to the $\mathrm{H}_{\delta 2}$ site. With these findings, we briefly discuss the electronic structure of dopamine in the context of UV and NMR measurements and then suggest possible relevance of our findings to dopamine function.

Absorption of UV and visible radiation is associated with excitation of electrons from a low energy level to a higher energy level. The larger the gap between energy levels, the more energy is needed to be absorbed to promote the electron into the higher energy levels. Thus, the frequency of absorbed electromagnetic radiation will be higher corresponding to shorter wavelength, as the two quantities are inversely related. As stated in section 3.1 , the $\lambda_{\text {peak }} \sim 288 \mathrm{~nm}$ determined by UV spectroscopy indicates an $n \rightarrow \pi^{*}$ transition that corresponds to the protonated nitrogen which harbors the extra valence electron of the chemical bond between the amine group and the benzene ring. The value of $\lambda_{\text {peak }}$ absorbed by DA indicates a relatively large energy gap for this transition. The nucleus is then less shielded due this large energy gap which affects the chemical shifts measured by NMR.

The chemical shift data in Fig. 2 describe the behavior of DA as a function of hydrophobicity levels. A clear downward trend of ppm values for each aromatic hydrogen indicates shielding, or increased electron density. Although there is no chemical reaction taking place, the interaction of 
DA and IPA increases as a function of increasing concentration of IPA, i.e. with increasing hydrophobicity. With the lowest measured ppm values (Fig. 2), the $\mathrm{H}_{\delta 1}$ site prefers a more hydrophobic environment consistent with the EIC map in Fig. 5. For all aromatic sites, the change in chemical shifts in Fig. 2 are relatively abrupt up to 30\% IPA where they appear to stabilize except for $\mathrm{H}_{\delta 1}$. This behavior can be rationalized by considering the hydration layers around dopamine: as isopropanol concentration increases, water molecules in the hydration shell of dopamine are progressively replaced by isopropanol either because of a preference of dopamine for isopropanol or simply because of entropic (mixing) reasons. Dehydration of dopamine also occurs when it binds to lipid bilayers, in this case water molecules being replaced by lipid and therefore affecting the measured NMR chemical shifts.

In future studies, it will be interesting to complement these solution NMR measurements with solid-state techniques such as ${ }^{31} \mathrm{P}$ and ${ }^{14} \mathrm{~N}$ NMR spectroscopy. Solid-state methods have been shown to provide information on how ions [14, 15], local anesthetics [15, 16], and polypeptides [17] affect the conformation and dynamics of lipid headgroups and are therefore applicable to studies of dopamine as well.

Our results with lipid vesicles suggest that the extent of dopamine release in the brain could depend on lipid composition. PS lipids in particular are directly involved in recognition of apoptotic cells and this can be relevant to impairment of dopamine-generating cells. DA infused lipid vesicles could potentially cause macrophage activity that could mitigate the spread of early stages of Parkinson's disease. In early stages it is possible to locate the aggregation of $\alpha$-Synuclein in dendrites (which causes Parkinson's) using an MRI scan. Injecting high DA loaded vesicles in these location can take advantage of the natural tendency of DA to leak out, travel through the axon terminal, and then pass through the synaptic cleft to the dendrite. Research shows an acceleration of apoptosis when DA directly binds to $\alpha$-Synuclein [18]. High concentrations of expelled DA could initiate apoptosis due to DNA fragmentation commenced by the body's response to the excess amounts of DA [19]. Consequently, apoptosis can trigger macrophage activity to engulf the infected dendrite and possibly halting the proliferation of Parkinson's to normal functioning dendrites. 


\subsection{REFERENCES}

1. R. G. Perez, J. C. Waymire, E. Lin, J. J. Liu, F. Guo, and M. J. Zigmond, A Role for $\alpha$-Synuclein in the Regulation of Dopamine Biosynthesis, J. Neurosci., 22 (2002) 3090-3099.

2. M. S. Sonders, S-J. Zhu, N. R. Zahniser, M. P. Kavanaugh, and S. G. Amara1, Multiple Ionic Conductances of the Human Dopamine Transporter: The Actions of Dopamine and Psychostimulants, J. Neurosci., 17 (1997) 960-974.

3. J. M. Musacchio, "Chapter 1: Enzymes involved in the biosynthesis and degradation of catecholamines", Edited by L. Iverson, Biochemistry of Biogenic Amines, Springer, 2014 pp. 1-35.

4. J. Fantini and N. Yahi, "Chapter 6: Protein-Lipid interactions in the brain" in Brain Lipids in Synaptic Function and Neurological Disease, Elsevier, 2015, pp. 135-162.

5. F. Benfenati, P. Greengard, J. Brunner, and M. Bähler, Electrostatic and hydrophobic interactions of synapsin I and synapsin I fragments with phospholipid bilayers, J. Cell Biol., 108 (1989) 1851-1862.

6. E. Schwarz, S. Prabakaran, P. Whitfield, H. Major, F.M. Leweke, D. Koethe, P. McKenna, and S. Bahn, High throughput lipidomic profiling of schizophrenia and bipolar disorder brain tissue reveals alterations of free fatty acids, phosphatidylcholines, and ceramides, J. Proteome Res., 7 (2008) 4266-77.

7. K. Farmer, C. A. Smith, S. Hayley, J. Smith Major Alterations of Phosphatidylcholine and Lysophosphotidylcholine Lipids in the Substantia Nigra Using an Early Stage Model of Parkinson's Disease, Int. J. Mol. Sci., 16 (2015) 18865-18877.

8. B. Verhoven, R. A Schlegel, P. Williamson. Mechanisms of phosphatidylserine exposure, a phagocyte recognition signal, on apoptotic T lymphocytes, J Exp. Med., 182, 1597-601, 2014. 
9. C. P. Müller, M. Reichel, C. Mühle, C. Rhein, E. Gulbins, J. Kornhuber, Brain membrane lipids in major depression and anxiety disorders, Biochim. Biophys. Acta, 1851 (2015) 1052-65.

10. M. A. Johnson, B. D. Ray, S. R. Wassall, and H.I. Petrache, Equivalent Isopropanol Concentrations of Aromatic Amino Acids Interactions with Lipid Vesicles, J. Membrane Biol, 248 (2015) 695-703.

11. T. López, J. Bata-García, D. Esquivel, E. Ortiz-Islas, R. Gonzalez, J. Ascencio, P. Quintana, G. Oskam, F. J. Álvarez-Cervera, F. J. HerediaLópez, and J. L Góngora-Alfaro, Treatment of Parkinson's disease: nanostructured sol-gel silica-dopamine reservoirs for controlled drug release in the central nervous system, Int. J. Nanomedicine, 6 (2011) 19-31.

12. I. Ziv, E. Melamed, N. Nardi, D. Luria, A. Achiron, D. Offen, A. Barzilai, Dopamine induces apoptosis-like cell death in cultured chick sympathetic neurons- A possible novel pathogenetic mechanism in Parkinson's disease, Neurosci Lett., 170 (1994) 136-40.

13. K. Jodko-Piorecka, and G. Litwinienko, First Experimental Evidence of Dopamine Interactions with Negatively Charged Model Biomembranes, ACS Chem. Neurosci., 4 (2013) 1114-1122.

14. H. I. Petrache, S. Tristram-Nagle, K. Gawrisch, D. Harries, V. A. Parsegian, and J. F. Nagle. Structure and Fluctuations of Charged Phophatidylserine Bilayers in the Absence of Salt. Biophys. J. 86 (2004) 1574-1586.

15. D. J. Siminovitch, M. F. Brown, and K. R. Jeffrey, 14N NMR of Lipid Bilayers: Effects of Ions and Anesthetics, Biochemistry 23, (1984) 24122420 .

16. J. S. Santos, D.-K. Lee, and A. Ramamoorthy, Effects of antidepressants on the conformation of phospholipid headgroups studies by solid-state NMR, Magn. Reson. Chem., 42 (2004) 105-114. 
17. J. P. F. Doux, B. A. Hall, and J. A. Killian, How lipid headgroups sense the membrane environment: An application of 14N NMR, Biophys. J. 103 (2012) 1245-1253.

18. F.J.S.Lee, F. Liu, Z. B. Pristupa, and H. B. Niznik, Direct binding and functional coupling of $\alpha$-synuclein to the dopamine transporters accelerate dopamine-induced apoptosis, FASEB J., 15 (2001) 916-926.

19. R. Simantov, E. Blinder, T. Ratovitski , M. Tauber, M. Gabbay, and S, Porat, Dopamine-induced apoptosis in human neuronal cells: inhibition by nucleic acids antisense to the dopamine transporter, Neuroscience, 74 (1996) 39-50. 


\section{Figures}

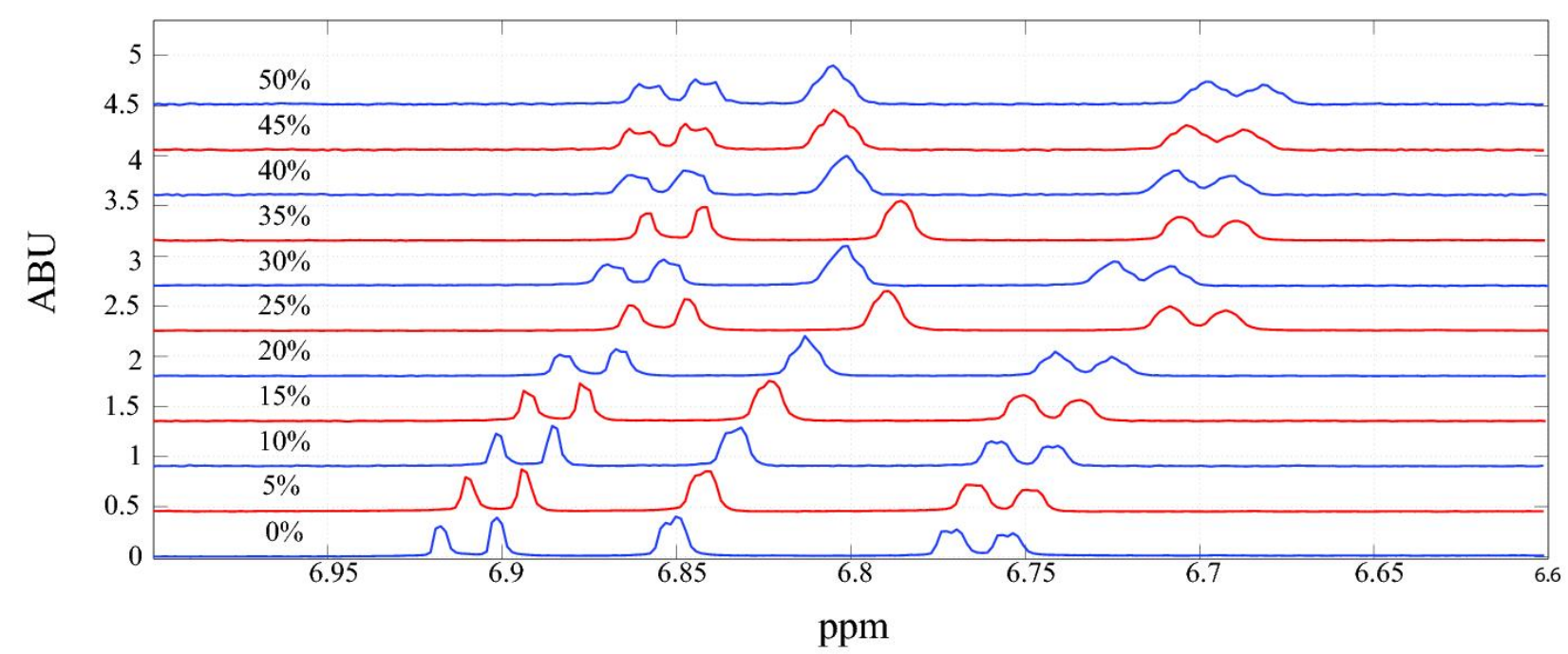

Figure 1. ${ }^{1} \mathrm{H}$ NMR spectra of dopamine in various concentrations of isopropanol. The spectra are shown in arbitrary units (ABU) and have been shifted vertically for clarity. 


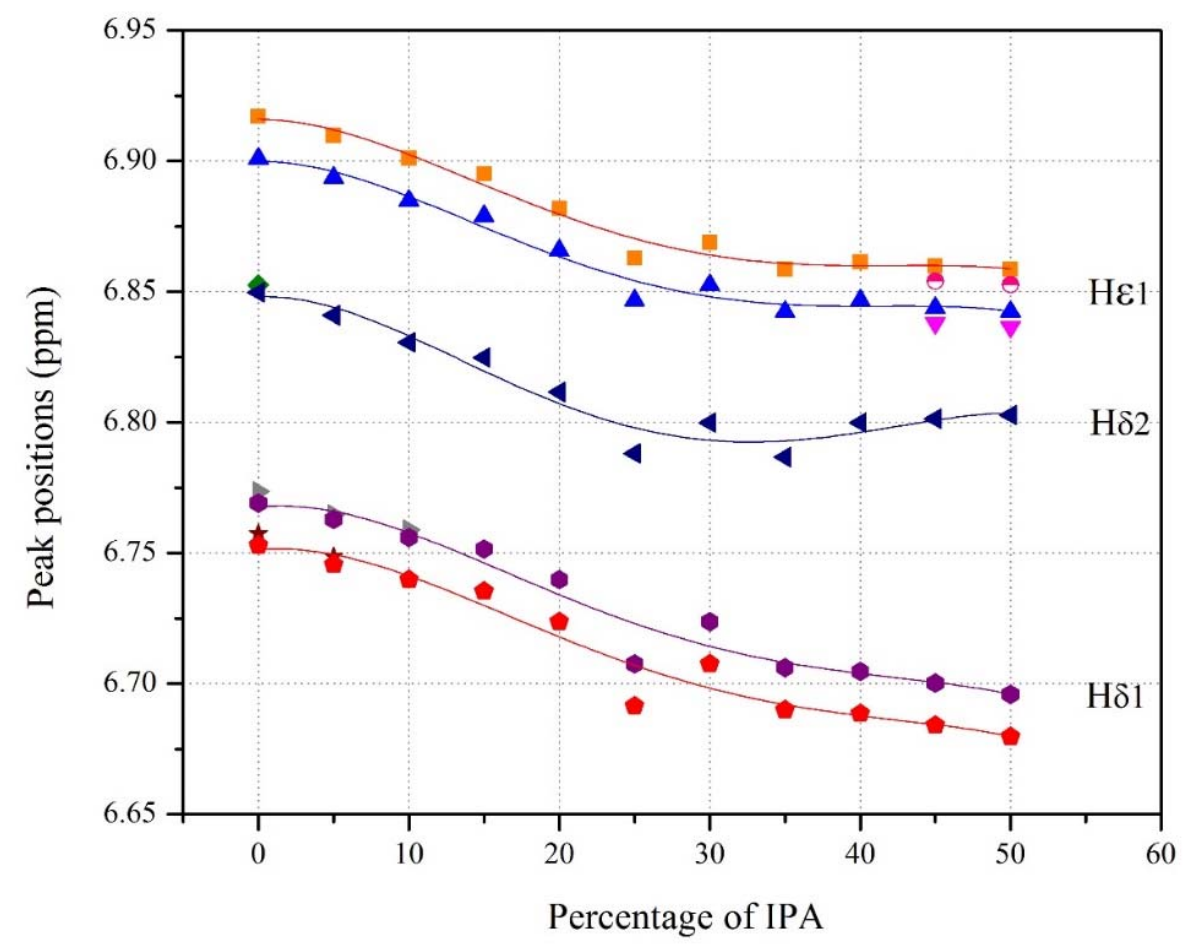

Figure 2. ${ }^{1} \mathrm{H}$ NMR chemical shifts of dopamine as a function of isopropanol concentration. Resonance assignments are indicated on the right side of the graph. 


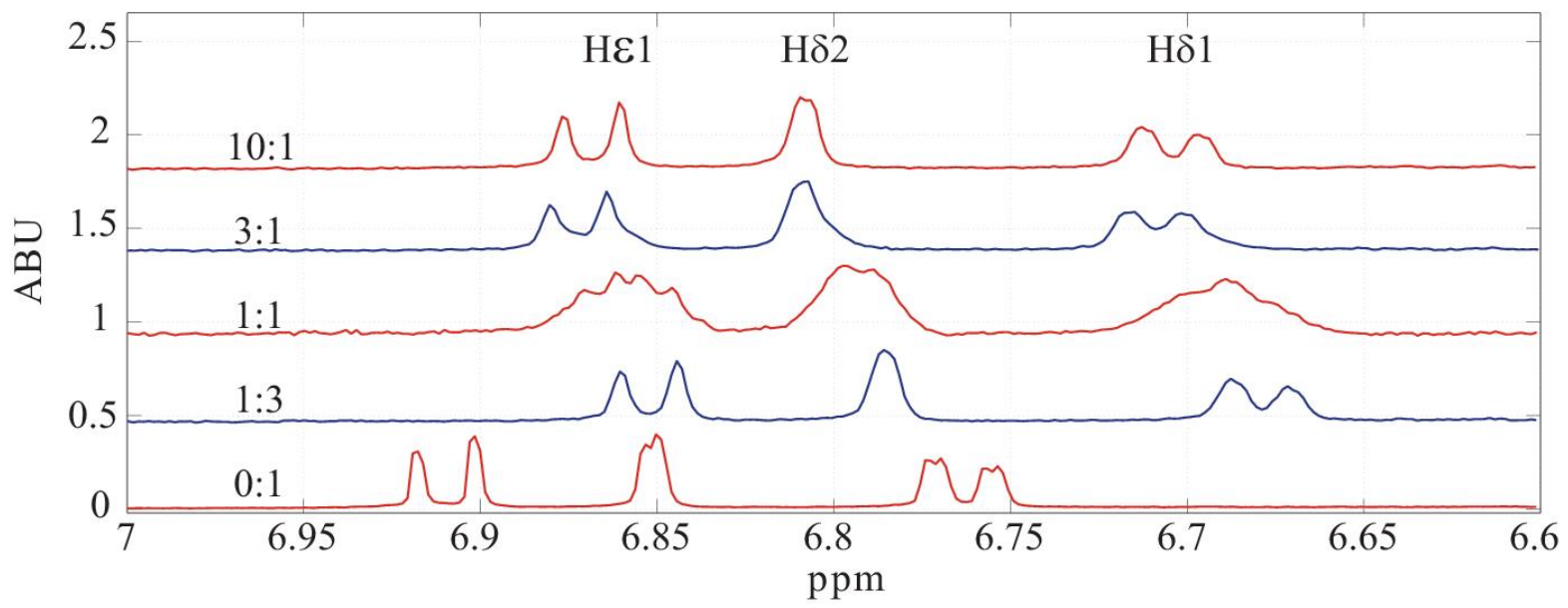

Figure 3. ${ }^{1} \mathrm{H}$ NMR spectra of dopamine in the presence of DOPC lipid vesicles at various lipid:dopamine molar ratios. The spectra are shown in arbitrary units $(\mathrm{ABU})$ and have been shifted vertically for clarity. 


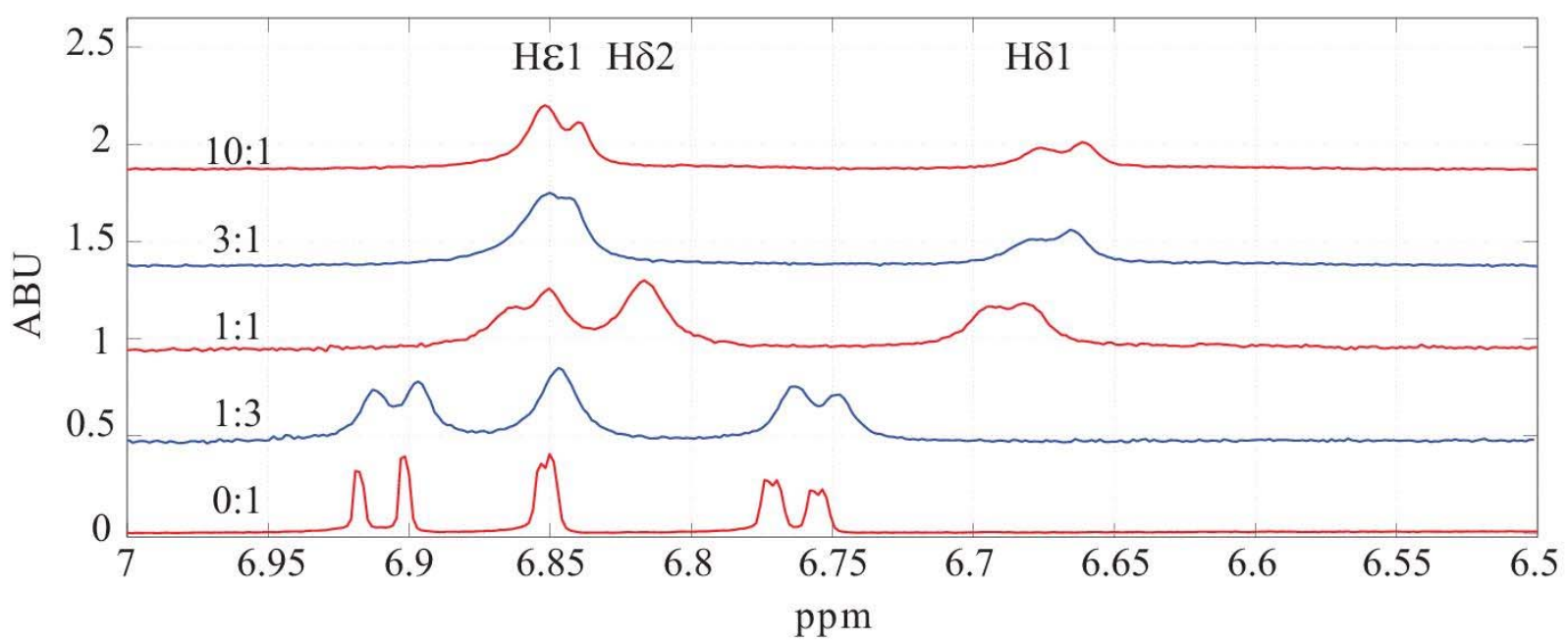

Figure 4. ${ }^{1} \mathrm{H}$ NMR spectra of dopamine in the presence of DOPS lipid vesicles at various lipid:dopamine molar ratios. The spectra are shown in arbitrary units (ABU) and have been shifted vertically for clarity. 

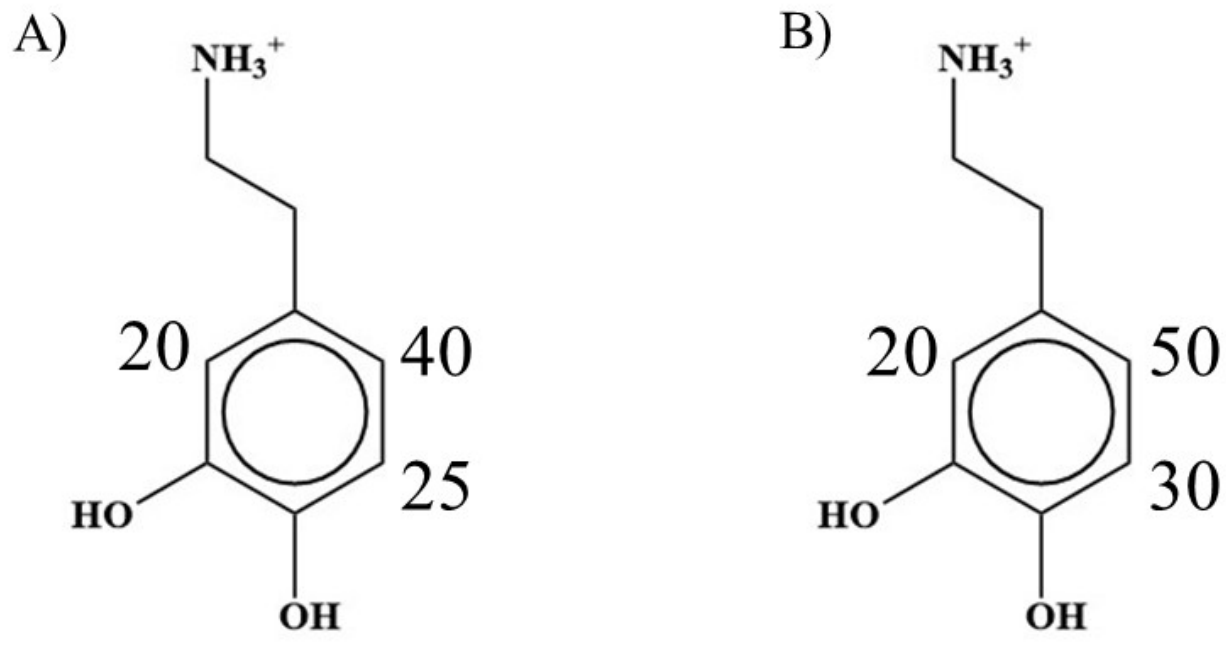

Figure 5. Equivalent Isopropanol Concentration (EIC) maps of dopamine interacting with PC lipids (panel A) and with PS lipids (panel B). 


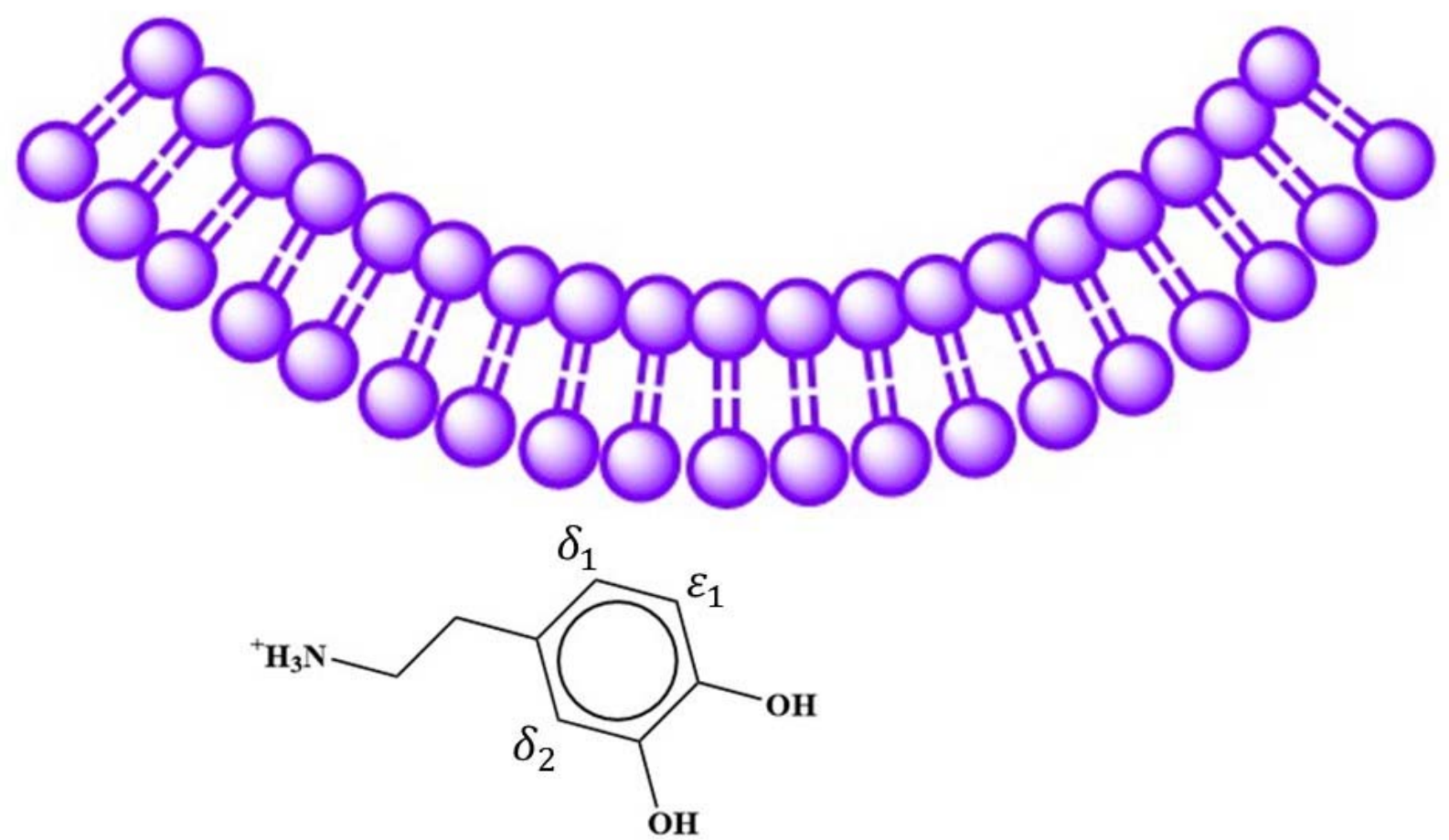

Figure 6. Schematics of dopamine orientation with respect to lipid bilayers based on the measured EIC values. 


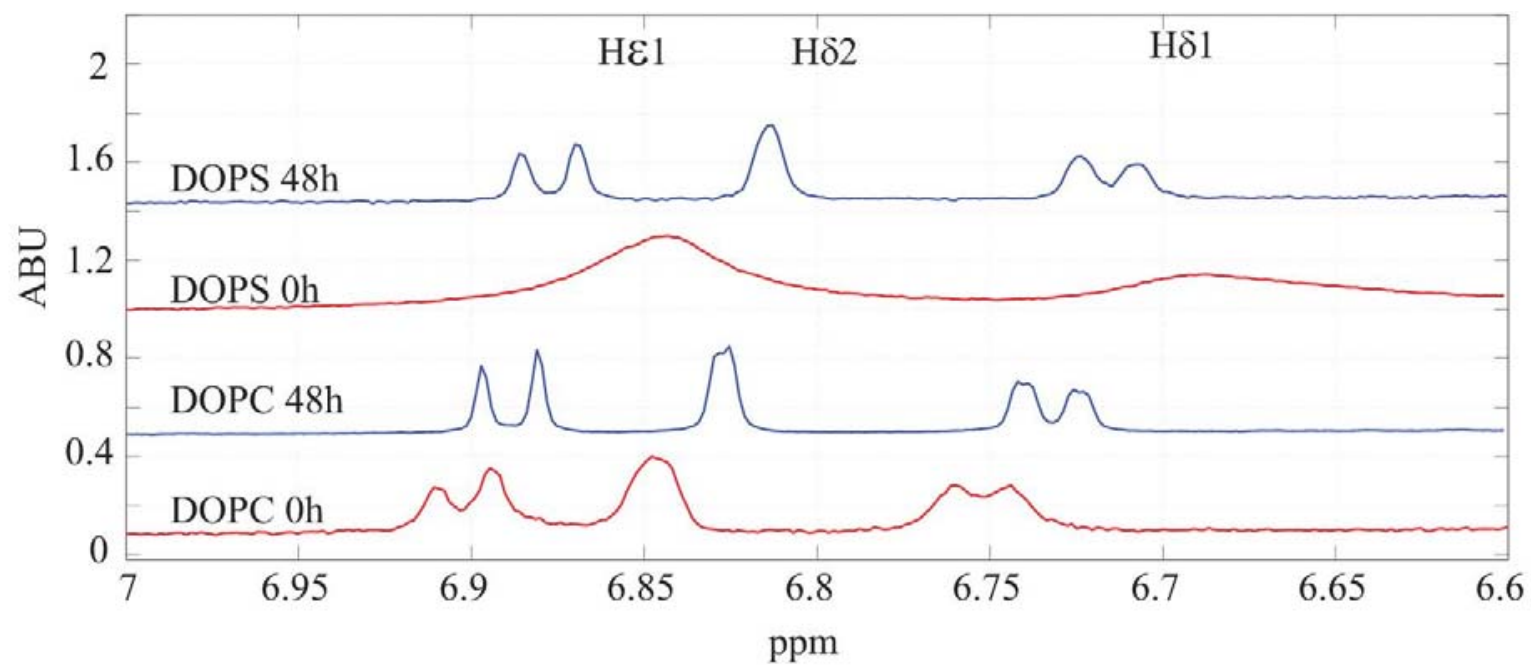

Figure 7. ${ }^{1} \mathrm{H}$ NMR spectra of dopamine trapped inside lipid vesicles (measurements at $0 \mathrm{~h}$ ) and leaked outside (measurements at $48 \mathrm{~h}$ ). The spectra are shown in arbitrary units (ABU) and have been shifted vertically for clarity. 